\title{
Role of Mindfuless in The Current World of Mental Health
}

\author{
Sreetama Chatterjee \\ Clinical Psychologist, Department of Clinical Psychology, Institute of Psychiatry, Kolkata
}

You can't stop the waves, but you can learn to surf" - Kabat-Zinn, 2004

\section{INTRODUCTION}

Perhaps one of the best advantages that Mental Health Professionals get to enjoy is that they are considered as healers, whose primary aim is to alleviate emotional suffering; whether be it through medication or through psychotherapy or through both. However, even after so many years of emergence of treatment of psychological complaints, we are now at a junction where the very above-mentioned statement stands corrected or at least questioned. That being, "Can we humans truly ever evade pain and suffering" or should we find another way by enabling ourselves to deal better with it? Needless to say, in order to answer this, one has to first look at the root cause of suffering and investigate what are the main psychological factors that cause and perpetuate suffering and poses threats to mental health. Now research has identified some psychological factors, such as the meanings people attach to cognitive phenomena in their own right, that is, cognition about cognition or "metacognition" [1] that can perpetuate psychological disorders like depression, anxiety disorders, psychophysiological disorders. At the same time, it has also been noted that although psychotherapy has come a long way in treating these symptoms, however the need for mindfulness has emerged over the years. In order to understand this better, perhaps a brief discussion on the different waves of therapy need to be briefly considered.

\section{$1^{\text {ST }}$ WAVE THERAPY}

Although, reportedly the first records of advocating psychotherapy for treatment of the "insane" is credited to Paracelsus back through hundreds of years, however it was not until the time of Sigmund Freud that psychotherapy experienced a major breakthrough. However, next significant change had occurred at around the 1950s, in the wake of the World War II, in 1940s which gave rise to need for an effective short term therapy for depression and anxiety.The surge in behavioral research which came about due to psychologists like Skinner, Wolpe , and Eysenck; primarily regarding how people "learn" to behave and react emotionally. (Behavioral learning theory) is what one knows commonly as the "first wave" of behavior

\section{$2^{\text {ND }}$ WAVE THERAPY}

The first wave was soon followed by a second revolution in the late 1960s wherein psychologists and psychiatrists began the empirical study of how cognitions and language affected emotions and behavior and highlighted their importance in treatment of psychopathology. This phase also known as second wave therapy had witnessed some groundbreaking developments like that of Ellis' rational emotive behavior therapy, and Beck and colleagues' cognitive therapy and indicated a shift more towards exploration of the ways in which one's interpretations of the world, especially the interpretation of emotionally relevant situations, shapes experience.

\section{$3^{\mathrm{RD}}$ WAVE THERAPY}

However, a lacuna in overall holistic treatment was still present even after the emergence of both the first and second wave therapies, since complete reduction of symptoms was difficult to achieve along with difficulty in maintaining the benefits post follow-up. In this regard, research showed that the preoccupation with change was one of the most salient points of why relapse keeps occurring. 
Thus, it was realized over time that if a therapy has to be truly successful, then it has to strive to change the patients' relationship to his/her experience to suffering, or how one can get less upset by events in their life ${ }^{[2]}$. Repeated research also showed how the interpretations people attach to symptoms they experience, can serve to maintain and exacerbate psychological problems ${ }^{[3]}$. This hence implied that, rather than seeking to alter the content of cognitions, at times it can be more helpful to reduce their unhelpful impact by changing the nature of the patient's relationship to them ${ }^{[2]}$. This is what paved the way for emergence of the third wave therapies in a sharp contrast to the therapies that have been applied before. These third wave therapies came to be defined as those which emphasize the acceptance of and contact with the present moment as a sole mean of achieving positive mental health and are also known as second order factor theories is the focus more on increasing the individual's capacities rather than focusing on symptom reduction. The main third wave therapies that emerged are :

- Mindfulness Based Stress Reduction or MBSR ${ }^{[4]}$

- Mindfulness based Cognitive Therapy or $\mathrm{MBCT}^{[5]}$

- Acceptance and Commitment Based therapies or $\mathrm{ACT}^{[6]}$

- Dialectical Behavior Therapy or $\mathrm{DBT}^{[7]}$

\section{MINDFULNESS - TERMS AND CONCEPTS}

Fundamental to all these approaches is a focus on developing mindfulness. The term 'mindfulness' originates from the 'Pali' language word 'sati' meaning 'to remember'[8]. Mindfulness has also defined as the awareness that emerges when we pay attention to experience in a particular way: on purpose (the attention is deliberately placed on particular aspects of experience); in the present moment (when the mind slips into the past or the future we bring it back to the present); and non judgmentally (the process is infused with a spirit of acceptance of whatever arises) $)^{[9]}$. It is also conceived as a form of experiential processing and refers to a "presence of mind" wherein attention, informed by a sensitive awareness of what is occurring in the present, simply observes what is taking place, whether external events or internal (psychological and somatic) experiences. This can be sharply contrasted with the conceptually driven mode of processing in which experience is habitually filtered through cognitive appraisals, evaluations, memories, beliefs, and other forms of cognitive manipulation ${ }^{[10]}$. This non- mindful state, also known as mindlessness or automatic pilot is usually characterized by getting entangled in distracting thoughts, rushing through activities without being attentive to them, finding oneself preoccupied with future or past.

In order to elaborate further, Kabat-Zinn [4] had delineated certain attitudinal qualities that underpin mindfulness practice which are stated in the following:

- Non-judging : assume an impartial witness to one's own experience

- Patience : cultivating patience by giving oneself room to have the experience, whether good or bad, because it is a part of reality.

- Trust : developing trust in self and honoring our feelings,

- Beginner's Mind : "to see the richness of the present moment by having a mind that is willing to see everything as if for the first time"

- Non-striving : Simply allowing anything to be experienced in each moment because it is there

- Acceptance : means seeing things as they actually are in the present and lastly

- Letting go : cultivating the attitude of letting go, or non-attachment by letting things be as they are, without judging or holding on.

Thus, it can be generally seen, that engaging in mindfulness practices leads to increased awareness which in turn leads to greater psychological and emotional freedom; and with close mindfulness if one can generate insight in the source of suffering 
or unpleasantness, then it might open new avenues for freeing from harmful mental reactions. But how the approach of mindfulness can help us in understanding the psychopathology of some of the most common psychiatric disorders and also help in dealing with them perhaps needs a little more elaboration.

\section{DEPRESSION}

In the course of research on depression, Beck ${ }^{[11]}$, had identified certain themes such as low self-regard, unrealistic downgrading/devaluation of the self and magnification of failures and defects as certain maintain factors. Other researchers had given a similar view that depression reflects a negative, selfrelated patterns of information-processing which becomes accessible in mild, normal depressed mood, and are then fuelled by experiential avoidance and self-devaluative rumination ${ }^{[12]}$. The mindfulness approach also hypothesized that, depressive patients are most likely to "turn away" from his or her experience, or what we know more commonly as "experiential avoidance"[6] and exhibit an 'abandonement ${ }^{[2]}$. Although research over the years have indicated both antidepressant medication and cognitive behavior therapy to be effective in treating depressive episodes, however some disadvantages have been deemed to both these treatments. That being, patients taking maintenancepharmacotherapy need to keep taking it for a long time for the beneficial aspects to sustain, and cognitive behavioral treatment relies on the person engaging in one-to one with a skilled, and scarce therapist. Also many a times maintaining the efficacy on follow up and post follow -up on cognitive behavior therapy has proven to be a difficult task. Additionally, in case of these patients, making them actively engaged in CBT also becomes very difficult ${ }^{[12]}$. Thus to address these issues, MBCT developed as an approach for treating people with a history of depression and who are therefore vulnerable to future episodes ${ }^{[5]}$. While in depression in mindfulness based approach such as MBCT, therapists help the patients to note where there has been a turning away, and then turn towards and be with the experience at hand. With an aim of assessing its efficacy. One important research had examined the effects of MBCT (a practice of mindfulness that draws heavily from MBSR and also from cognitive behavioral scientific and therapeutic principles ) on rates of depressive and their results showed much lower relapse rates for patients with three or more previous depressive episodes, even during the year follow-up period ${ }^{[13]}$. This finding was later replicated by other researches which indicates that mindfulness based approaches have been deemed useful both in conceptualizing its pathology and also in its treatment.

\section{ANXIETY}

The Mindfulness based approaches believe that much human suffering results from the counterproductive habit of avoiding painful situations ${ }^{[2]}$. Thus, from this perspective, anxiety disorders are developed when individuals are not willing to experience anxiety, which may come in various forms like thoughts, emotions and related emotions, with which they are struggling; and in order to deal with the anxiety, a person engages in various internal as well as external control strategies. Significant behavioral as well experiential avoidance is thus developed and generalized to avoid the unwanted mental states elicited by events or objects which are feared. As this avoidance behavior is increased, person may begin to make changes that have significant impact on his or her quality of life. Some researchers believe that attempts at avoidance only serve to perpetuate distress. Also, essentially what all anxiety disorders have in common is intolerance for the experience of anxiety. So some researchers believe that it might also be sustained by engaging in negative metacognitions, that is thinking about negative mental events ${ }^{[14]}$.

Hence, the main tools used in treating anxiety with the help of mindfulness based therapies are: awareness and exposure. In this context, awareness refers to gaining insight about the transitory nature of thoughts, by viewing them as just a mental event and accepting it, rather than trying to fight with it or avoid it. Thus treatment involves gradually turning 
patient's attention toward the fear as it is happening, exploring the fear in detail with increasing degrees of friendly acceptance. This leads to more moment to moment awareness which helps in dismantling the fear by distinguishing the raw facts of experience from the frightening conclusions that are drawn from it ${ }^{[2]}$. Research has suggested that mindfulness practice may be efficacious through increasing meta-cognitive awareness, a cognitive set associated with "decentering" or "disindentification," in which negative thoughts and feelings are experienced as mental events rather than as the self. Thus there is gradual shift in the patient's relationship to anxiety from avoidance to tolerance to friendship.

Researchers have also found promising results of mindfulness based approaches in treating anxiety ${ }^{[15]}$ had found that an 8 week group intervention based on the mindfulness meditation (MBSR), had led to significant reduction in anxiety and depression among individuals meeting the criteria for generalized anxiety and panic disorders which was maintained after three years as well.

\section{PSYCHOPHYSIOLOGICAL DISORDERS}

As the quote in the beginning of the chapter said, pain is one aspect of life that nobody can escape, yet no one really likes it. Instead one always makes frantic efforts to get rid of it. Ironically, research has shown that a remarkable variety of medical disorders are actually maintained by our attempts to feel better. For instance, some of the most common psychophysiological disorders which have been effected by this vicious cycle are chronic back pain, and other muscle and joint disorders, such as tendonitis, plantar fasciitis, temporomandibular joint syndrome, chronic headache and fibromyalgia. Although many of these disorders are caused by structural damage, injury, or disease processes, yet they are also perceived as muscle tension disorders. Many a times, the cycle might begin with physical/psychological stress, and once the patient becomes concerned and starts focusing on the painful area, he also starts protecting it and abandons all normal activity. This paves the way for worry and frustration, and the pain cycle becomes established. Likewise, the distress associated with gastrointestinal, dermatological disorders, sexual dysfunction, and insomnia can also be attributed to a large extent to psychological stress ${ }^{[2]}$.

Thus, this implies a large scope for mindfulness based interventions in this domain and research in this area has in fact shown great promise. The proposed rationale behind the success of mindfulness based approaches in this regard, could be attributed to the fact that mindfulness practice essentially helps us to develop s a more accepting, kind and tolerant attitude towards the symptoms, as well as to increase emotional awareness and to relax counterproductive attempts at control ${ }^{[2]}$.

\section{THE BENEFITS OF MINDFULNESS BASED THERAPIES}

Meta-analytic studies have shown over time a clear support for the efficacy of mindfulness on physical and mental well being. For instance in a meta-analytic study by Baer ${ }^{[16]}$ mindfulness based interventions were proven highly effective for treating patients with Axis I disorders such as anxiety and depression, global psychological symptoms, medical disorders such as fibromyalgia, psoriasis and even cancer. Later, another study ${ }^{[17]}$ had also found similar results in their meta-analytic study. However some studies have indicated otherwise, where the effectiveness was brought into question on basis of some methodological flaws. Speaking of various forms of therapies based on mindfulness, one study comparing the effectiveness of DBT, with treatment as usual for patients with BPD showed that Dialectical Behaviour Therapy resulted in better retention rates and greater reductions of self-mutilating and selfdamaging impulsive behaviours compared with usual treatment, especially among those with a history of frequent self-mutilation ${ }^{[18]}$. Additionally, DBT has also shown decreases on mean self-rated depression scores in clients suffering from major depression. Additionally, Meta-analyses of ACT, 
yet another variant of mindfulness based approach has shown it to be more effective than placebo for most problems, with the exception of anxiety and depression, but no more effective than traditional therapies like cognitive behavioral therapy. Another meta-analysis reported that ACT outperformed CBT, except for depression and anxiety ${ }^{[19]}$. In fact most recently, a 2015 meta-analysis found that ACT was more effective than placebo and "treatment as usual", and as effective as traditional treatments like CBT for depression, anxiety disorders, addiction and somatic health problems ${ }^{[20]}$

\section{CONCLUSION}

Thus to conclude, it can be said that we are now possibly at the helm of a new era of mental health, where the concept of mindfulness characterized by kind acceptance, has shown major breakthroughs, however further research is needed to assess its efficacy measures on a larger basis and in the long term. But if the statistics are to be believed, then it sure seems that the benefits of engaging in mindfulness are here to stay

\section{REFERENCE}

Wells, A. Emotional disorders and metacognition: Innovative cognitive therapy. Chichester, UK : Wiley; 2000.

Germer, C.K., Siegel., R.D., Fulton, P.R. editors. Mindfulness and Psychotherapy. New York : Guilford; 2005.

Fennell, M.J.V. Depression, low self-esteem and mindfulness. Behavior Research and Therapy. 2004 : 42 : 1053-1067.

Kabat-Zinn, J.Full Catastrophe Living: How to cope with stress, pain and illness using mindfulness meditation. New York : Dell Publishing; 1990.

Segal, Z. V., Williams, J. M. G., Teasdale, J. D. (2002). Mindfulnessbased cognitive therapy for depression: A new approach to preventing relapse. New York: Guilford; 2002.

Hayes, S.C., Strosahl, K.D., Wilson, K.G. Acceptance and commitment therapy: An experiential approach to behavior change. New York : Guilford; 1999.
Linehan, M. Skills training manual for treating borderline personality disorder. New York : Guilford; 1993b.

Bodhi, B. A comprehensive manual of Abhidhamma.Seattle, WA : BPS Pariyatti Editions; 2000.

Kabat-Zinn, J. Mindfulness meditation for everyday life. New York Hyperion;1994.

Brown, K.W., Ryan, R.M., \& Creswell, J.D. Mindfulness : Theoretical Foundations and Evidence for its salutary effects. Psychological Inquiry. 2007 : 18, 211-237.

Beck, A. T. Depression : Clinical, experimental and theoretical aspects. New York : Harper \& Row; 1967.

Crane, R. Mindfulness - Based Cognitive Therapy Distinctive Features. Great Britain: Routledge; 2009.

Teasdale, J. D., Segal, Z. V., Williams, J. M. G., Ridgeway, V., Soulsby, J., \& Lau, M. Prevention of relapse/recurrence in major depression by mindfulness-based cognitive therapy. Journal of Consulting and Clinical Psychology. 2000 : 68, 615-623.

Toneatto, T. A metacognitive therapy for anxiety disorders. Buddhist psychology applied. Cognitive and Behavioral Practice. 2002 : 9(1), 72-7.

Kabat-Zinn, J., Massion, M. D., Kristeller, J. Electiveness of a meditation-based stress reduction program in the treatment of anxiety disorders. American Journal of Psychiatry. 1992 : 149, 936-943.

Baer, R.A. Mindfulness training as a clinical intervention : A conceptual and empirical review. Clinical Psychology : Science and Practice. 2003 : 10, 125-143.

Grossman,P.,Niemann, L., Schmidt, S., \&Walach, H. Mindfulnessbased stress reduction and health benefits. A meta-analysis. Psychosomatic Research. 2004 : 57, 35-43.

Verheul, R., V.D.B., L. M., Koeter, M.W., De Ridder, M.A., Stinjen, T.,Van Den Brink, W. Dialectical Behavior Therapy for women with borderline personality disorder 12-month, randomized clinical trial in Netherlands. The British Journal of Psychiatry. 2003 : 182 (2), 135-140.

Ruiz, F.J. Acceptance and commitment therapy versus traditional cognitive behavioral therapy: A systematic review and meta-analysis of current empirical evidence. International Journal of Psychology and Psychological therapy. 2012 : 12, 333-358.

Davis, M.L., Morina., N., Powers, M.B., Smits, J. A.J , Emmelkamp, P.M.G. A meta analysis of the efficacy of acceptance and commitment therapy for clinically relevant mental and physical health problems. Psychotherapy and Psychosomatics. 2015 : 84(1), 30-36. 